\title{
NONLINEAR FILTERING WITH CORRELATED NOISES IN INFINITE DIMENSION
}

\author{
C. BOULANGER* ${ }^{*}$, J. SCHILTZ ${ }^{\dagger}$ \\ U.R.A. C.N.R.S. No 399, Département de Mathématiques, Université de Metz, \\ BP 80794, F-57012 Metz Cedex \\ * Fax : 0387315273 and e-mail : boulange@poncelet.univ-metz.fr \\ † Fax : 0387315273 and e-mail : schiltz@poncelet.univ-metz.fr
}

Keywords Nonlinear filtering, Stochastic differential equation, Stochastic calculus.

\begin{abstract}
In this paper, we derive the Kushner-Stratonovich and the Zakai equation for the filter and the unnormalized filter associated with a nonlinear filtering problem with correlated noises, bounded coefficients and a signal process evolving in an infinite dimensional space. A robust form of the Zakai equation is established when the noises are independent.
\end{abstract}

\section{Introduction}

The purpose of this paper is to prove that the unnormalized filter associated with a nonlinear filtering problem with correlated noises, bounded coefficients and a signal process evolving in an infinite dimensional space, solves the Zakai equation. Then, a Kushner-Stratonovich equation for the filter is deduced by usual arguments from the Kallianpur-Striebel formula.

This problem has already been investigated when the signal process is finite dimensional by many authors. M. Zakai [13] has showed in the case of independent noises, that the unnormalized filter is, if it exists, solution of a stochastic partial differential equation of parabolical type. Then, M. Davis [3], M. Davis and S. I. Marcus [4] and E. Pardoux [11] have extended Zakai's method to the case of nonlinear filtering problems with correlated noises.

As we know, infinite dimensional stochastic processes, solutions of a stochastic differential equation driven by infinite dimensional Brownian motions, are related with certain continuous state Ising-type models in statistical mechanics, and also with models arising in population genetics. In [5], P. Florchinger has proved that the unnormalized filter associated with a nonlinear filtering problem with independent noises and bounded coefficients solves a
Zakai equation, even if the signal process is infinite dimensional.

This paper is divided in six sections organized as follows. In the second section, we introduce the nonlinear filtering problem studied in this paper and we show that it has a unique strong solution with a.s. continuous paths. In section three, we define an unnormalized filter linked with the filter defined in the previous section by means of a Kallianpur-Striebel formula. In the fourth and fifth sections we derive the Zakai and the Kushner-Stratonovich equations associated with our nonlinear filtering problem. The sixth section, finally, is devoted to deduce a robust form of the Zakai equation under the hypothesis that the noises are independent.

\section{Setting of the problem}

Let $(\Omega, \mathcal{F}, P)$ be a complete probability space and $\gamma=$ $\left\{\gamma_{i}, i \in \mathbb{Z}\right\}$ a summable sequence of strictly positive real numbers.

Set

$$
\begin{aligned}
& L^{2}(\gamma)=\left\{x=\left(x_{i}\right) \in \mathbb{R}^{\mathbb{Z}}:\|x\|_{\gamma}^{2}=\sum_{i \in \mathbb{Z}} \gamma_{i}\left|x_{i}\right|^{2}<+\infty\right\}, \\
& L^{2}(\gamma \times \gamma)=\left\{x=\left(x_{j}^{i}\right) \in \mathbb{R}^{\mathbb{Z} \times \mathbb{Z}}:\right. \\
& \left.\qquad\|x\|_{\gamma \times \gamma}^{2}=\sum_{i, j \in \mathbb{Z}} \gamma_{i} \gamma_{j}\left|x_{j}^{i}\right|^{2}<+\infty\right\} \\
& \text { and } L^{2}(\gamma \times p)=\left\{x=\left(x_{k}^{i}\right) \in \mathbb{R}^{\mathbb{Z} \times p}:\right. \\
& \left.\qquad\|x\|_{\gamma \times p}^{2}=\sum_{i \in \mathbb{Z}} \sum_{k=1}^{p} \gamma_{i}\left|x_{k}^{i}\right|^{2}<+\infty\right\}
\end{aligned}
$$

On the other hand, consider the nonlinear filtering problem associated with the signal-observation pair $\left(x_{t}, y_{t}\right) \in$ $L^{2}(\gamma) \times \mathbb{R}^{p}$ solution of the stochastic differential system 


$$
\left\{\begin{array}{c}
x_{t}^{i}=x_{0}^{i}+\int_{0}^{t} b_{i}\left(s, x_{s}\right) d s+\int_{0}^{t} \sum_{j \in \mathbb{Z}} \sigma_{j}^{i}\left(s, x_{s}\right) d w_{s}^{j} \\
+\sum_{k=1}^{p} g_{k}^{i}\left(s, x_{s}\right) d v_{s}^{k}, \quad i \in \mathbb{Z} \\
y_{t}=\int_{0}^{t} h\left(s, x_{s}\right) d s+v_{t},
\end{array}\right.
$$

where,

1. $W=\left\{w_{t}^{i}, t \in[0, T], i \in \mathbb{Z}\right\}$ is a family of independent Brownian motions with variances $\gamma_{i} t$.

2. $V=\left\{v_{t}, t \in[0, T]\right\}$ is a p-dimensional standard Brownian motion independent of $W$.

3. $x_{0}$ is an $L^{2}(\gamma)$-valued random variable independent of $W$ and $V$.

4. The maps $b:[0, T] \times \mathbb{R}^{\mathbb{Z}} \rightarrow \mathbb{R}^{\mathbb{Z}}, \sigma:[0, T] \times \mathbb{R}^{\mathbb{Z}} \rightarrow$ $\mathbb{R}^{\mathbb{Z} \times \mathbb{Z}}$ and $g:[0, T] \times \mathbb{R}^{\mathbb{Z}} \rightarrow \mathbb{R}^{\mathbb{Z} \times p}$ are such that there exists a strictly positive constant $K$ for which $\left(H_{1}\right) \quad \forall t \in[0, T], \forall x \in L^{2}(\gamma)$,

$$
\begin{aligned}
\|b(t, x)\|_{\gamma}^{2}+\|\sigma(t, x)\|_{\gamma \times \gamma}^{2}+\|g(t, x)\|_{\gamma \times p}^{2} & \\
& \leq K\left(1+\|x\|_{\gamma}^{2}\right)
\end{aligned}
$$

and

$$
\begin{aligned}
& \left(H_{2}\right) \quad \forall t \in[0, T], \forall x, y \in L^{2}(\gamma), \\
& \|b(t, x)-b(t, y)\|_{\gamma}^{2}+\|\sigma(t, x)-\sigma(t, y)\|_{\gamma \times \gamma}^{2} \\
& +\|g(t, x)-g(t, y)\|_{\gamma \times p}^{2} \leq K\|x-y\|_{\gamma}^{2} .
\end{aligned}
$$

5. $h:[0, T] \times \mathbb{R}^{\mathbb{Z}} \rightarrow \mathbb{R}^{p}$ is a bounded Lipschitz function with less than linear growth.

6. The maps $b, \sigma$ and $g$ are uniformly bounded.

Then, the stochastic differential system (1) is well defined for the stochastic processes $x$ in $L^{2}\left(\Omega \times[0, T] ; L^{2}(\gamma)\right)$ and $y$ in $L^{2}\left(\Omega \times[0, T] ; \mathbb{R}^{p}\right)$. Moreover, if $u=\left\{u_{t}^{i}, t \in\right.$ $[0, T], i \in \mathbb{Z}\}$ is an $L^{2}(\gamma)$-valued square integrable and adapted process, we have, (cf. [10]) :

$$
E\left[\left(\int_{0}^{t} \sum_{i \in \mathbb{Z}} u_{s}^{i} d w_{s}^{i}\right)^{2}\right]=E\left(\int_{0}^{t} \sum_{i \in \mathbb{Z}} \gamma_{i}\left|u_{s}^{i}\right|^{2} d s\right),
$$

which allows us to prove the following existence and unicity theorem :

Theorem 2.1 For any $L^{2}(\gamma)$-valued random variable $x_{0}$ independent of the Brownian motion $\left\{W_{t}, t \in[0, T]\right\}$, the stochastic differential system (1) has a unique continuous $L^{2}(\gamma)$-valued strong solution $\left\{x_{t}, t \in[0, T]\right\}$ such that $E\left(\sup _{t \in[0, T]}\left\|x_{t}\right\|_{\gamma}^{2}\right)<+\infty$.

\section{proof :}

We use Picard's iteration method to construct an approximation of the solution of (1). Set

$$
\left\{\begin{aligned}
x_{t}^{(0)}=x_{0} & \\
x_{t}^{i(n+1)}= & x_{0}^{i}+\int_{0}^{t} b_{i}\left(s, x_{s}^{(n)}\right) d s \\
& +\int_{0}^{t} \sum_{j \in \mathbb{Z}} \sigma_{j}^{i}\left(s, x_{s}^{(n)}\right) d w_{s}^{j} \\
& +\int_{0}^{t} \sum_{k=1}^{p} g_{k}^{i}\left(s, x_{s}^{(n)}\right) d v_{s}^{k},
\end{aligned}\right.
$$

for each $n \geq 0$.

At first, we show by induction on $n$, that

$E\left\{\sup _{t \in[0, T]}\left\|x_{t}^{(n)}\right\|_{\gamma}^{2}\right\}<+\infty$, to ensure that (3) makes sense. Indeed, the relation (2), as well as the Minkovski and Burkholder inequalities and condition $\left(H_{1}\right)$ imply

$$
\begin{aligned}
E\left\{\sup _{t \in[0, T]}\left\|x_{t}^{(n+1)}\right\|_{\gamma}^{2}\right\} \leq & C\left(\sum _ { i \in \mathbb { Z } } \left\{\gamma_{i} E\left|x_{0}^{i}\right|^{2}\right.\right. \\
& +E \int_{0}^{T} \gamma_{i}\left|b_{i}\left(s, x_{s}^{(n)}\right)\right|^{2} d s \\
& +E \int_{0}^{T} \sum_{j \in \mathbb{Z}} \gamma_{i}\left|\sigma_{j}^{i}\left(s, x_{s}^{(n)}\right)\right|^{2} d s \\
& \left.\left.+E \int_{0}^{T} \sum_{k=1}^{p} \gamma_{i}\left|g_{k}^{i}\left(s, x_{s}^{(n)}\right)\right|^{2} d s\right\}\right) \\
\leq & C_{1}+C_{2} E\left\{\sup _{t \in[0, T]}\left\|x_{t}^{(n)}\right\|_{\gamma}^{2}\right\} \\
\leq & \ldots \leq E\left\{\sup _{t \in[0, T]}\left\|x_{t}^{(0)}\right\|_{\gamma}^{2}\right\}<+\infty
\end{aligned}
$$

On the other hand, the same arguments and condition $\left(H_{2}\right)$ show that

$$
\begin{aligned}
E\left\{\sup _{t \in[0, T]}\right. & \left.\left\|x_{t}^{(n+1)}-x_{t}^{(n)}\right\|_{\gamma}^{2}\right\} \\
& \leq C E \int_{0}^{T}\left\|x_{s}^{(n)}-x_{s}^{(n-1)}\right\|_{\gamma}^{2} d s .
\end{aligned}
$$

Consequently, the sequence $\left(x^{(n)}\right)_{n \geq 0}$ is a Cauchy sequence in the complete space $L^{2}\left(\Omega \times[0, T] ; L^{2}(\gamma)\right)$, so it possesses a limit, which ensures the existence of an a.s. continuous process $x=\left\{x_{t}, t \in[0, T]\right\}$ such that

$E\left\{\sup _{t \in[0, T]}\left\|x_{t}^{(n)}-x_{t}\right\|_{\gamma}^{2}\right\} \rightarrow 0, \quad$ when $n \rightarrow+\infty$.

Furthermore it is easy to see that $x$ verifies (1).

Now, let us prove now the uniqueness of the solution.

If $\tilde{x}=\left\{\tilde{x}_{t}, t \in[0, T]\right\}$ denotes another solution of (1), the 
same arguments that we have used to prove (4) imply that $E\left\{\sup _{t \in[0, T]}\left\|x_{t}-\tilde{x}_{t}\right\|_{\gamma}^{2}\right\} \leq C \int_{0}^{T} E\left[\sup _{0 \leq s \leq t}\left\|x_{s}-\tilde{x}_{s}\right\|_{\gamma}^{2}\right] d t$.

Therefore the uniqueness of the solution follows from Gronwall's lemma.

Moreover, since $x_{t}^{(n)}$ tends almost surely to $x_{t}$, we get by Fatou's lemma that $E\left\{\sup _{t \in[0, T]}\left\|x_{t}\right\|_{\gamma}^{2}\right\}<+\infty$.

To determine the infinitesimal generator associated with the stochastic process $\left\{x_{t}, t \in[0, T]\right\}$, denote for all $t \in[0, T], x \in L^{2}(\gamma), i, j \in \mathbb{Z}$ and $k=1, \ldots, p$ the matrices $a(t, x)$ and $\alpha(t, x)$ in $\mathcal{M}_{\mathbb{Z} \times \mathbb{Z}}(\mathbb{R})$ and $\mathcal{M}_{\mathbb{Z} \times p}(\mathbb{R})$ respectively, defined by

and

$$
a_{j}^{i}(t, x)=\sum_{l \in \mathbb{Z}} \gamma_{l} \sigma_{l}^{i}(t, x) \sigma_{l}^{j}(t, x)
$$

$$
\alpha_{k}^{i}(t, x)=\sum_{l=1}^{p} \gamma_{l} g_{l}^{i}(t, x) g_{l}^{k}(t, x) .
$$

Notice that under the condition $\left(H_{2}\right)$, the matrices $a(t, x)$ and $\alpha(t, x)$ satisfy the following property :

For every strictly positive constant $C$, there exists a strictly positive constant $K_{C}$ such that, for any $t \in[0, T]$,

$$
\begin{aligned}
\|a(t, x)-a(t, y)\|_{\gamma \times \gamma}^{2}+\|\alpha(t, x)-\alpha(t, y)\|_{\gamma \times p}^{2} & \\
& \leq K_{C}\|x-y\|_{\gamma}^{2},
\end{aligned}
$$

for all $x, y \in L^{2}(\gamma)$ such that $\|x\|_{\gamma}^{2}$ and $\|y\|_{\gamma}^{2}$ are less than $C$.

Notation 2.2 Denote by $\mathcal{D}^{2}$ the set of functions $f$ : $[0, T] \times \mathbb{R}^{\mathbb{Z}} \rightarrow \mathbb{R}$ such that there exists $M \in \mathbb{N}^{*}$, a subset $\left\{i_{1}, \ldots, i_{M}\right\} \subset \mathbb{Z}$ and a $\mathcal{C}_{0}^{1,2}$-function $\bar{f}:[0, T] \times \mathbb{R}^{M} \rightarrow \mathbb{R}$ such that $f(t, x)=\bar{f}\left(t, x_{i_{1}}, \ldots, x_{i_{M}}\right)$ for every $t \in[0, T]$ and $x \in \mathbb{R}^{\mathbb{Z}}$.

Hence, we have :

Proposition 2.3 (cf. [10]) The process $\left\{x_{t}, t \in[0, T]\right\}$, solution of the stochastic differential system (1) is a Markov diffusion process whose infinitesimal generator $L$ is defined for every function $f$ in $\mathcal{D}^{2}$ by

$$
\begin{aligned}
L f(t, x)= & \frac{\partial}{\partial t} f(t, x)+\sum_{i \in \mathbb{Z}} b_{i}(t, x) \nabla_{i} f(t, x) \\
& +\frac{1}{2} \sum_{i, j \in \mathbb{Z}} a_{j}^{i}(t, x) \nabla_{i, j} f(t, x) \\
& +\frac{1}{2} \sum_{i \in \mathbb{Z}} \sum_{k=1}^{p} \alpha_{k}^{i}(t, x) \nabla_{i, k} f(t, x) .
\end{aligned}
$$

Then, as usually in nonlinear filtering theory we then define the filter associated with (1) by
Definition 2.4 For all $t \in[0, T]$, denote by $\Pi_{t}$ the filter associated with the stochastic differential system (1), defined for every function $\psi$ from $[0, T] \times \mathbb{R}^{\mathbb{Z}}$ to $\mathbb{R}$ by

$$
\Pi_{t}(\psi)=E\left[\psi_{t}\left(t, x_{t}\right) / \mathcal{Y}_{t}\right]
$$

where $\mathcal{Y}_{t}=\sigma\left(y_{s} / 0 \leq s \leq t\right)$.

Remark : We could have defined the filter for a larger class of functions, but we have restricted it here to functions in $\mathcal{D}^{2}$, because the Zakai and Kushner-Stratonovich equations are only valid for such functions.

\section{The reference probability mea- sure}

In order to define an unnormalized filter, we make use of the "reference probability measure" method to transform the stochastic process $\left\{y_{t}, t \in[0, T]\right\}$ into a standard Wiener process. With this aim set

Definition 3.1 For all $t$ in $[0, T]$, denote by $Z_{t}$ the Girsanov exponential defined by

$Z_{t}=\exp \left(\int_{0}^{t} \sum_{k=1}^{p} h_{k}\left(s, x_{s}\right) d v_{s}^{k}+\frac{1}{2} \int_{0}^{t}\left|h\left(s, x_{s}\right)\right|^{2} d s\right)$.

Since $\left(Z_{t}\right)_{t \in[0, T]}$ is a martingale, we can introduce the reference probability measure as follows.

Definition 3.2 Denote by $\bar{P}$ the reference probability measure, defined on the space $\left(\Omega, \mathcal{F}, \mathcal{F}_{t}\right)$ by the RadonNikodym derivative

$$
\frac{d \bar{P}}{d P} / \mathcal{F}_{t}=Z_{t}^{-1}
$$

Then, by Girsanov's theorem, the process $\left\{y_{t}, t \in[0, T]\right\}$ is a standard Brownian motion on the space $\left(\Omega, \mathcal{F}, \mathcal{F}_{t}, \bar{P}\right)$ independent of the Wiener process $W$.

Hence we can define the unnormalized filter associated with the system (1) in the following way

Definition 3.3 For all $t$ in $[0, T]$, denote by $\rho_{t}$ the unnormalized filter associated with the stochastic differential system (1), defined for every function $\psi$ from $[0, T] \times \mathbb{R}^{\mathbb{Z}}$ in $\mathbb{R}$ by

$$
\rho_{t} \psi=\bar{E}\left[\psi\left(t, x_{t}\right) Z_{t} / \mathcal{Y}_{t}\right],
$$

where $\bar{E}$ denotes the expectation with under the probability $\bar{P}$.

Furthermore, we have the following Kallianpur - Striebel formula which links the filter and the unnormalized filter.

Proposition 3.4 (cf. [9] or [12]) For all $t$ in [0,T] and every function $\psi$ in $\mathcal{D}^{2}$, we have

$$
\Pi_{t} \psi=\frac{\rho_{t} \psi}{\rho_{t} 1} .
$$




\section{The Zakai equation}

In this section, we show that the unnormalized filter $\rho_{t}$ defined by (7) solves a Zakai equation associated to the system (1). For this, we need the following stochastic Fubini theorem :

Lemma 4.1 (cf. [7]) If $U_{t}$ is a stochastic process such that $\bar{E} \int_{0}^{t} U_{s}^{2} d s<+\infty$, then

$$
\bar{E}\left[\int_{0}^{t} \sum_{i \in \mathbb{Z}} U_{s}^{i} d w_{s}^{i} / \mathcal{Y}_{t}\right]=0
$$

and

$$
\bar{E}\left[\int_{0}^{t} \sum_{k=1}^{p} U_{s}^{k} d y_{s}^{k} / \mathcal{Y}_{t}\right]=\int_{0}^{t} \sum_{k=1}^{p} \bar{E}\left[U_{s}^{k} / \mathcal{Y}_{t}\right] d y_{s}^{k} .
$$

Theorem 4.2 For every function $\psi$ in $\mathcal{D}^{2}$, the unnormalized filter $\rho_{t}$ is a solution of the stochastic partial differential equation

$\rho_{t}(\psi)=\rho_{0}(\psi)+\int_{0}^{t} \rho_{s}(L \psi) d s+\int_{0}^{t} \sum_{k=1}^{p} \rho_{s}\left(L_{k} \psi\right) d y_{s}^{k}$,

where $L_{k}$ is the first order differential operator defined for any function $\psi$ in $\mathcal{D}^{2}$ by

$$
L_{k} \psi(t, x)=\sum_{i \in \mathbb{Z}} g_{k}^{i}(t, x) \nabla_{i} \psi(t, x)+h_{k}(t, x) \psi(t, x) .
$$

proof :

By Itô's formula,

$$
\begin{aligned}
d \psi\left(t, x_{t}\right)= & L \psi\left(t, x_{t}\right) d t+\sum_{i, j \in \mathbb{Z}} \sigma_{j}^{i}\left(t, x_{t}\right) \nabla_{i} \psi\left(t, x_{t}\right) d w_{t}^{j} \\
& +\sum_{i \in \mathbb{Z}} \sum_{k=1}^{p} g_{k}^{i}\left(t, x_{t}\right) \nabla_{i} \psi\left(t, x_{t}\right) d v_{t}^{k}
\end{aligned}
$$

Hence,

$$
\begin{aligned}
d\left(\psi\left(t, x_{t}\right) Z_{t}\right)= & L \psi\left(t, x_{t}\right) Z_{t} d t \\
& +\sum_{i, j \in \mathbb{Z}} \sigma_{j}^{i}\left(t, x_{t}\right) Z_{t} \nabla_{i} \psi\left(t, x_{t}\right) d w_{t}^{j} \\
& +\sum_{i \in \mathbb{Z}} \sum_{k=1}^{p} g_{k}^{i}\left(t, x_{t}\right) Z_{t} \nabla_{i} \psi\left(t, x_{t}\right) d v_{t}^{k} \\
& +\sum_{k=1}^{p} Z_{t} h_{k}\left(t, x_{t}\right) \psi\left(t, x_{t}\right) d y_{t}^{k} \\
& +\sum_{i \in \mathbb{Z}} \sum_{k=1}^{p} g_{k}^{i}\left(t, x_{t}\right) h_{k}\left(t, x_{t}\right) \nabla_{i} \psi\left(t, x_{t}\right) Z_{t} d t \\
= & L \psi\left(t, x_{t}\right) Z_{t} d t
\end{aligned}
$$

$$
\begin{aligned}
& +\sum_{i, j \in \mathbb{Z}} \sigma_{j}^{i}\left(t, x_{t}\right) \nabla_{i} \psi\left(t, x_{t}\right) Z_{t} d w_{t}^{j} \\
& +\sum_{k=1}^{p} L_{k}\left(\psi\left(t, x_{t}\right)\right) Z_{t} d y_{t}^{k} .
\end{aligned}
$$

Consequently,

$$
\begin{aligned}
\rho_{t}(\psi)= & E\left[\psi\left(0, x_{0}\right)\right]+\int_{0}^{t} \bar{E}\left[L \psi\left(s, x_{s}\right) Z_{s} / \mathcal{Y}_{s}\right] d s \\
& +\sum_{k=1}^{p} \int_{0}^{t} \bar{E}\left[L_{k}\left(\psi\left(s, x_{s}\right)\right) Z_{s} / \mathcal{Y}_{s}\right] d y_{s}^{k} .
\end{aligned}
$$

\section{The Kushner - Stratonovich equation}

In this section, we prove that the filter $\Pi_{t}$ defined by (5) solves a Kushner-Stratonovich equation. With this aim, we show at first the following result by applying Itô's formula to the process $Z_{t}$.

Proposition 5.1 For all $t$ in $[0, T]$,

$\rho_{t} 1=\exp \left(\int_{0}^{t} \sum_{k=1}^{p} \Pi_{s}\left(h_{k}\right) d y_{s}^{k}-\frac{1}{2} \int_{0}^{t} \sum_{k=1}^{p}\left(\Pi_{s}\left(h_{k}\right)\right)^{2} d s\right)$.

Again, by applying Itô's formula to the processes $\left(\rho_{t} 1\right)^{-1}$ and $\rho_{t} \psi\left(\rho_{t} 1\right)^{-1}$ we proof the following Kushner Stratonovich formula :

Theorem 5.2 For all $t$ in $[0, T]$ and every function $\psi$ in $\mathcal{D}^{2}$, the filter $\Pi_{t}(\psi)$ is the solution of the stochastic differential equation

$$
\begin{aligned}
\Pi_{t}(\psi) & =\Pi_{0}(\psi)+\int_{0}^{t} \Pi_{s}(L \psi) d s \\
+ & \int_{0}^{t} \sum_{k=1}^{p}\left[\Pi_{s}\left(L_{k} \psi\right)-\Pi_{s}\left(h_{k}\right) \Pi_{s}(\psi)\right]\left[d y_{s}^{k}-\Pi_{s}\left(h_{k}\right)\right] d s .
\end{aligned}
$$

\section{The robust form of the Zakai equation}

In this section, we derive the robust form of the Zakai equation (8) obtained previously. The robust form of the Zakai equation has been introduced by J. Clark [2] to define a "robust" filter associated with a noncorrelated filtering system with bounded observation coefficients. This method allows to turn up the resolution of an Itô type stochastic differential equation to an ordinary partial differential equation parametrized by the paths of the observation process. W. Hopkins [8] then established, by 
means of an analoguous method, a robust form for the Zakai equation for a noncorrelated nonlinear filtering system with unbounded observation coefficients. Since, in the case of a multidimensional observation process, this method is however only adapted to the case of a noncorrelated filtering problem, we shall suppose in this section that $g \equiv 0$, so we consider the system process-observation pair $\left(x_{t}, y_{t}\right) \in L^{2}(\gamma) \times \mathbb{R}^{p}$ solution of the stochastic differential system

$$
\left\{\begin{array}{l}
x_{t}^{i}=x_{0}^{i}+\int_{0}^{t} b_{i}\left(s, x_{s}\right) d s+\int_{0}^{t} \sum_{j \in \mathbb{Z}} \sigma_{j}^{i}\left(s, x_{s}\right) d w_{s}^{j}, \\
i \in \mathbb{Z} \\
y_{t}=\int_{0}^{t} h\left(s, x_{s}\right) d s+v_{t} .
\end{array}\right.
$$

Remark: In the case of a one-dimensional observation process, M. Davis [3], respectively J.M. Bismut and D. Michel [1], have derived a robust form for the Zakai equation for a correlated nonlinear filtering system with bounded coefficients, whereas P.Florchinger [6] proved a similar result for a correlated nonlinear filtering system with unbounded observation coefficients.

Definition 6.1 For every $t$ in $[0, T]$ and every function $\psi$ in $\mathcal{D}^{2}$, set

$$
\nu_{t} \psi=\bar{E}\left[\psi\left(t, x_{t}\right) U_{t} / \mathcal{Y}_{t}\right]
$$

where $U_{t}$ is the stochastic process defined by

$U_{t}=\exp \left(-\int_{0}^{t} \sum_{k=1}^{p} y_{s}^{k} d h_{k}\left(s, x_{s}\right)-\frac{1}{2} \int_{0}^{t} \sum_{k=1}^{p}\left(h_{k}\left(s, x_{s}\right)\right)^{2} d s\right)$.

Besides, by an integration by parts in the stochastic integral appearing in the formula (6), we get

$$
\begin{gathered}
Z_{t}=\exp \left(<h\left(t, x_{t}\right), y_{t}>-\int_{0}^{t} \sum_{k=1}^{p} y_{s}^{k} d h_{k}\left(s, x_{s}\right)\right. \\
\left.-\frac{1}{2} \int_{0}^{t} \sum_{k=1}^{p}\left(h_{k}\left(s, x_{s}\right)\right)^{2} d s\right),
\end{gathered}
$$

which allows us to deduce that for any function $\psi$ in $\mathcal{D}^{2}$, we have

$$
\nu_{t} \psi=\rho_{t}\left(\psi \exp \left(-<h\left(t, x_{t}\right), y_{t}>\right)\right) .
$$

With this, we can prove the following theorem :

Theorem 6.2 For every function $\psi$ in $\mathcal{D}^{2}, \nu_{t} \psi$ solves the ordinary partial differential equation:

$$
\left\{\begin{array}{c}
\frac{d}{d t} \nu_{t} \psi=\nu_{t}\left(L_{y_{t}} \psi\right) \\
\nu_{0} \psi=E\left[\psi\left(0, x_{0}\right)\right],
\end{array}\right.
$$

where $L_{y_{t}}$ is the second order partial differential operator, parametrized by the paths of the process $y$, defined for any function $\psi$ in $\mathcal{D}^{2}$ by
$L_{y_{t}} \psi(t, x)=L \psi(t, x)$

$$
\begin{aligned}
& -\sum_{i, j \in \mathbb{Z}} \sum_{k=1}^{p}\left(\sigma_{j}^{i}(t, x)\right)^{2} \nabla_{i} h_{k}(t, x) \nabla_{i} \psi(t, x) y_{t}^{k} \\
& +\left(-\frac{1}{2} \sum_{k=1}^{p}\left(h_{k}(t, x)\right)^{2}\right. \\
& -\sum_{k=1}^{p} L h_{k}(t, x) y_{t}^{k} \\
& \left.+\frac{1}{2} \sum_{i, j \in \mathbb{Z}} \sum_{k=1}^{p}\left(\sigma_{j}^{i}(t, x) \nabla_{i} h_{k}(t, x)\right)^{2} y_{t}^{k}\right) \psi(t, x) .
\end{aligned}
$$

proof :

According to Itô's formula,

$$
\begin{aligned}
U_{t}= & \exp \left(-\int_{0}^{t} \sum_{k=1}^{p} y_{s}^{k} L h_{k}\left(s, x_{s}\right) d s\right. \\
& -\frac{1}{2} \int_{0}^{t} \sum_{k=1}^{p}\left(h_{k}\left(s, x_{s}\right)\right)^{2} d s \\
& \left.-\int_{0}^{t} \sum_{i, j \in \mathbb{Z}} \sum_{k=1}^{p} \sigma_{j}^{i}\left(s, x_{s}\right) \nabla_{i} h_{k}\left(s, x_{s}\right) y_{s}^{k} d w_{s}^{j}\right) .
\end{aligned}
$$

Further applications of Itô's formula give

$$
\begin{aligned}
d U_{t}= & -\sum_{k=1}^{p} U_{t} L h_{k}\left(t, x_{t}\right) y_{t}^{k} d t \\
& -\frac{1}{2} \sum_{k=1}^{p} U_{t}\left(h_{k}\left(t, x_{t}\right)\right)^{2} d t \\
& -\sum_{i, j \in \mathbb{Z}} \sum_{k=1}^{p} U_{t} \sigma_{j}^{i}\left(t, x_{t}\right) \nabla_{i} h_{k}\left(t, x_{t}\right) y_{t}^{k} d w_{t}^{j} \\
& +\frac{1}{2} \sum_{i, j \in \mathbb{Z}} \sum_{k=1}^{p} U_{t}\left(\sigma_{j}^{i}\left(t, x_{t}\right) \nabla_{i} h_{k}\left(t, x_{t}\right) y_{t}^{k}\right)^{2} d t,
\end{aligned}
$$

and

$$
\begin{aligned}
d(\psi( & \left.\left.t, x_{t}\right) U_{t}\right)=L \psi(t, x) U_{t} d t \\
& +\sum_{i, j \in \mathbb{Z}} \sigma_{j}^{i}\left(t, x_{t}\right) \nabla_{i} \psi\left(t, x_{t}\right) U_{t} d w_{t}^{j} \\
& -\sum_{k=1}^{p} \psi\left(t, x_{t}\right) U_{t} y_{t}^{k} L h_{k}\left(t, x_{t}\right) d t \\
& -\frac{1}{2} \sum_{k=1}^{p} \psi\left(t, x_{t}\right) U_{t}\left(h_{k}\left(t, x_{t}\right)\right)^{2} d t \\
& -\sum_{i, j \in \mathbb{Z}} \sum_{k=1}^{p} \psi\left(t, x_{t}\right) U_{t} \sigma_{j}^{i}\left(t, x_{t}\right) \nabla_{i} h_{k}\left(t, x_{t}\right) y_{t}^{k} d w_{t}^{j} \\
& +\frac{1}{2} \sum_{i, j \in \mathbb{Z}} \sum_{k=1}^{p} \psi\left(t, x_{t}\right) U_{t}\left(\sigma_{j}^{i}\left(t, x_{t}\right) \nabla_{i} h_{k}\left(t, x_{t}\right) y_{t}^{k}\right)^{2} d t \\
& -\sum_{i, j \in \mathbb{Z}} \sum_{k=1}^{p} U_{t}\left(\sigma_{j}^{i}\left(t, x_{t}\right)\right)^{2} \nabla_{i} h_{k}\left(t, x_{t}\right) \nabla_{i} \psi\left(t, x_{t}\right) y_{t}^{k} d t
\end{aligned}
$$

The conclusion is then straightforward by means of definition 6.1 and lemma 4.1. 


\section{References}

[1] Bismut J.M., Michel D., Diffusions conditionnelles, Part I: J. Funct. Anal, 44, 174-211, (1981). Part II: J. Funct. Anal, 45, 274-292, (1982).

[2] Clark J., The design of a robust approximation to the stochastic differential equations of nonlinear filtering, in : Swirzynski J. (ed.), Communication systems and random process theory, Sijthoff and Nordhoof (1978).

[3] Davis M., Pathwise nonlinear filtering, in : Hazenwinkel and Willems, (eds.), Stochastic systems the mathematics of filtering and identification and applications, Reidel, Dordrecht, (1981).

[4] Davis M., Marcus S. I., An introduction to nonlinear filtering, in : Hazenwinkel and Willems, (eds.), Stochastic systems the mathematics of filtering and identification and applications, Reidel, Dordrecht, (1981).

[5] Florchinger P., Zakai equation of nonlinear filtering in infinite dimension, Proceedings of the 30th Conference on Decision and Control, 2754-2755, (1991).

[6] Florchinger P., Zakai equation of nonlinear filtering with unbounded coefficients. The case of dependent noises. System \& Control Letters, 21, 413-422, (1993).
[7] Holley R., Stroock D., Diffusions on an infinite dimensional torus, J. Funct. Anal., 42, 29-63, (1981).

[8] Hopkins W., Nonlinear filtering of non degenerate diffusions with unbounded coefficients, Thesis, University of Maryland at College Park, (1982).

[9] Kallianpur G., Stochastic filtering theory, Springer, (1980).

[10] Millet A., Nualart D., Sanz M., Time reversal for infinite dimensional diffusions, Probab. Theor. Rel. Fields, 82, 315-347, (1989).

[11] Pardoux E., Stochastic partial differential equations and filtering of diffusion processes, Stochastics, 3, 127-167, (1979).

[12] Pardoux E., Filtrage non linéaire et équations aux dérivées partielles stochastiques associées, in : Ecole d'été des Probabilités de Saint-Flour, Lect. Notes Math., 1464, Springer : Berlin, Heidelberg, NewYork, 69-163, (1989).

[13] Zakai M., On the optimal filtering of diffusion processes, Z. Wahrscheinlichkstheor. Verw. Gebiete, 11, 230-243, (1969). 